\title{
Abrupt Visual Onsets and Selective Attention: Voluntary Versus Automatic Allocation
}

\author{
Steven Yantis \\ Johns Hopkins University
}

\author{
John Jonides \\ University of Michigan
}

\begin{abstract}
The hypothesis that abrupt visual onsets capture attention automatically, as suggested by Yantis and Jonides (1984) was tested in four experiments. A centrally located cue directed attention to one of several stimulus positions in preparation for the identification of a target letter embedded in an array of distractor letters. In all experiments, one stimulus (either the target or one of the distractors) had an abrupt onset; the remaining letters did not. The effectiveness of the cue was manipulated (varying either its duration or its predictive validity) to test whether abrupt onsets capture attention even when subjects are in a highly focused attentional state. Results showed that onsets do not necessarily capture attention in violation of an observer's intentions. A mechanism for partially automatic attentional capture by abrupt onset is proposed, and the diagnosticity of the intentionality criterion for automaticity is discussed.
\end{abstract}

Introspective and empirical evidence both suggest that the abrupt appearance of an object in the visual field "draws attention." A plausible account of this phenomenon is that there exists a mechanism that is tuned to abrupt onsets and that one of its functions is to direct visual attention to the locus of an abrupt onset. This in turn could result in the efficient identification of information at that location. Another way of stating the hypothesis is that abrupt onsets may capture visual attention automatically and cause the observer to process abrupt visual events with high priority.

This hypothesis has two components. The first is that there is a mechanism that detects abrupt onsets and signals the visual attention system to allocate attentional resources to events exhibiting abrupt onsets. The second is that the allocation of attention resulting from such a signal is automatic. We examine each of these components in turn. The central thesis of this article is that although attention may be efficiently allocated to abrupt onset under some circumstances, this may not happen in a truly automatic fashion, as defined by widely held criteria. The implication of this thesis is either that attentional capture by abrupt onset is not automatic or that one of the commonly cited criteria for automaticity is not really diagnostic of automaticity.

This research was supported in part by funds from National Institute of Mental Health Grant RO1-MH43924 and from BRSG Grant SO7-RR07041 awarded by the Biomedical Research Support Grant Program, National Institutes of Health, to Steven Yantis and in part by Grant 82-0297 from the Air Force Office of Scientific Research to John Jonides.

We are grateful to Seema Chaudhari, Alyson Holoubek, and Scott Tebo for technical assistance. We thank Doug Johnson and James C. Johnston for valuable comments concerning the research, and James Cutting, John Duncan, Jeff Miller, Roger Remington, and an anonymous reviewer for suggestions and comments on an earlier version of this article.

Correspondence concerning this article should be addressed to Steven Yantis, Department of Psychology, Johns Hopkins University, Baltimore, Maryland 21218.

\section{Attentional Capture by Abrupt Onset}

The existence of a mechanism that may subserve attentional capture by abrupt onset is supported by evidence from several sources (see Yantis \& Jonides, 1984, for a more complete review). Electrophysiological (e.g., Cleland, Levick, \& Sanderson, 1973; Lennie, 1980) and psychophysical (e.g., Breitmeyer \& Ganz, 1976; Tolhurst, 1975) results have revealed visual mechanisms that are selectively sensitive to the abrupt onset and offset of visual stimuli, relative to their sustained presence. However, although some investigators (e.g., Breitmeyer \& Ganz, 1976) have speculated that channels sensitive to abrupt onset may subserve the allocation of visual attention, additional evidence is required to establish the functional relationship between these mechanisms and attention allocation.

Several kinds of evidence may be brought to bear on this functionality question. First, Todd and Van Gelder (1979) compared responses to stimuli that had abrupt onsets with responses to stimuli that did not. Rather than using gradual onsets as a control condition, as some other investigators had done, Todd and Van Gelder developed what they called the no-onset procedure. No-onset stimuli are presented by illuminating the stimuli in advance of the target display but camouflaging them with irrelevant line segments; the camouflaging segments are then removed, which reveals the noonset stimuli. Thus, the stimulus contours themselves are present before the stimulus is presented, and no localized abrupt onset accompanies their appearance (see Figures 1 and 3 for examples). The no-onset procedure is preferable to the use of gradual onset in reaction time experiments, because it permits one to anchor the appearance of stimuli precisely in time, as required for meaningful reaction time measurements. This is not possible with gradual-onset stimuli, or at least it is not possible without additional, complicating assumptions about detection and identification processes.

Todd and Van Gelder (1979) found that onset stimuli were detected more rapidly than were no-onset stimuli in tasks requiring rapid eye movement responses. The magnitude of the advantage for onset stimuli increased with the complexity 
of the decision that was required (from detection to categorization). Krumhansl (1982) reported data that confirmed and extended this result.

One account of these findings, advanced by Yantis and Jonides (1984), holds that abrupt onset captures attention automatically, which results in more favorable performance for onset versus no-onset stimuli. This hypothesis was supported by the results of a visual search experiment in which a prespecified target letter was to be detected in an array of two or four items. (Jonides and Yantis, 1988, replicated the results with display sizes of three, five, and seven stimuli, verifying the linearity of the display-size functions.) Every search display contained one onset stimulus and one or more no-onset stimuli. When the target was present (as it was on half of all trials), it was either an onset or a no-onset stimulus.

The critical measurements in this experiment concerned the effect of display size on reaction time as a function of whether the target was an onset or a no-onset stimulus. Standard visual search experiments yield linearly increasing reaction time functions of display size. When target-present functions have slopes that are roughly half those of targetabsent functions, the increase is usually interpreted as reflecting a serial, self-terminating search of the display. ${ }^{1}$ Flat display-size functions obtained under other conditions (e.g., Egeth, Jonides, \& Wall, 1972; Schneider \& Shiffrin, 1977; Treisman \& Gelade, 1980) typically are thought to reflect parallel visual search. Yantis and Jonides (1984) reasoned that if onsets capture attention automatically, this should be reflected in the slopes of the display-size functions: Reaction time should not increase with display size for onset targets, but it should increase in the usual serial, self-terminating fashion when the target is not an onset item.

The results were consistent with this hypothesis. Onset targets were evidently processed first regardless of their identity or position, and the data were quite well fit by a model in which the remaining stimuli were then scanned in a serial, self-terminating search. That attentional capture was automatic in this task was supported by the finding that reaction time did not increase with display size when the target had an abrupt onset while there was a significant slope when the target was of the no-onset type. As predicted by the selfterminating search model, the slope of the display-size function when the target was a no-onset stimulus was about half that of the function when the target was absent. The capture model provided a superior quantitative fit to the data than did several competing models.

Although Yantis and Jonides (1984) attributed capture by abrupt onset to properties of the visual system that are differentially sensitive to abrupt onset, it was nevertheless possible that the mere presence of a unique stimulus feature was the cause of attentional capture. According to this argument, any sufficiently salient and unique stimulus feature could yield the same result. Recently, Jonides and Yantis (1988) tested this idea by comparing the attention-capturing ability of abrupt onset with two other salient stimulus properties: color and intensity. In these experiments, the ability of an odd item (unique in either color or intensity) to capture attention was compared with the corresponding ability of an onset item. Only abrupt stimulus onset yielded the pattern of results characteristic of attentional capture. The results were inconsistent with the uniqueness account. Instead, it appears that abrupt onset has a privileged status in capturing attention.

The evidence reviewed in this section suggests that abrupt onsets draw attention and result in rapid identification of onset stimuli compared with no-onset stimuli. That attentional capture by abrupt onset may be automatic is supported by satisfaction of a load criterion for automaticity. In the next section, we examine more closely some of the criteria commonly applied to diagnose automaticity in visual search processes.

\section{Criteria for Automaticity}

Treatments of automatic information processing typically identify two properties that consistently accompany what are thought to be automatic processes (e.g., Hasher \& Zacks, 1979; Logan, 1978; Posner \& Snyder, 1975; Regan, 1981; Schneider \& Fisk, 1982; Schneider \& Shiffrin, 1977). Only if both of these criteria are satisfied is a process said to exhibit strong automaticity. The first criterion is insensitivity to concurrent perceptual or cognitive load: An automatic process is not hindered when concurrent information load is increased (we refer to this as the load-insensitivity criterion). The second criterion is that an automatic process is not subject to voluntary control: Attempts by a subject to prevent an automatic process from proceeding are not successful. We refer to this as the intentionality criterion (see Jonides, Naveh-Benjamin, \& Palmer, 1985; Palmer \& Jonides, 1988, for further discussion of these criteria).

Kahneman and Treisman (1984) defined three levels of automaticity in perception. A process is strongly automatic if it is not facilitated by focusing attention on a stimulus and not inhibited by focusing attention away from it. Thus a strongly automatic process satisfies both the load insensitivity criterion and the intentionality criterion. If either of these criteria is at least sometimes violated, a process is said to be partially or occasionally automatic (or, of course, not automatic at all). The Stroop effect is an example of a process (word recognition) that is normally completed even when attention is diverted from the stimulus but can be facilitated by allocating attention to it; word recognition is therefore a partially automatic process, according to Kahneman and Treisman. As discussed later, because the load-insensitivity and intentionality criteria are not inseparable, these shades of meaning influence the conclusions that are justified by our data.

As we stated above, in our previous work we speculated that abrupt onset may capture attention automatically. However, although we have found supporting evidence for the

\footnotetext{
${ }^{1}$ There are exceptions and complications associated with the interpretation of linear display-size functions in visual search (see, e.g., Townsend \& Ashby, 1983). These complications do not materially affect our argument and are therefore beyond the scope of the present discussion. We refer to linearly increasing display-size functions with 2:1 (negative:positive) slope ratios as reflecting serial, self-terminating search, with the understanding that such an interpretation is not the only one possible.
} 
load-insensitivity criterion, converging evidence for strong automaticity has not yet been obtained for the intentionality criterion. In the experiments reported below, we tested the intentionality criterion to determine whether attentional capture by abrupt onset may be characterized as strongly automatic.

A related effort was reported by Jonides (1981), who examined the extent to which a peripheral visual cue elicits an automatic shift of attention to the spatial location it indicates. He tested three criteria for automaticity and compared peripheral and central cues under each criterion. He found that cues appearing in the peripheral visual field capture attention automatically according to all three tested criteria. In particular, Jonides (1981) found in his Experiment 1 that peripheral cues produced attentional benefits regardless of whether a concurrent memory load was imposed on the subject whereas the effectiveness of a central cue was severaly attenuated under a concurrent memory load. This satisfied the load-insensitivity criterion. In his Experiment 2, Jonides discovered that even when peripheral cues were randomly associated with a visual search target and subjects were told to ignore the cues, reaction time was significantly faster when the cue was valid than when it was not. In contrast, subjects were able to ignore central cues that were only randomly related to the target position. A third experiment verified this resistance to subjects' intentions and expectancies evidenced by peripheral cues. Experiments 2 and 3 together provided evidence satisfying the intentionality criterion for automaticity. In summary, the results reported by Jonides (1981) are consistent with the hypothesis that peripheral visual events capture attention automatically.

Posner and Cohen (1984) subsequently showed that with short intervals between the cue and target events, attention appears to be summoned to the cue, but that at longer intervals there is a relative inhibition in detecting events at the cued location. Posner and Cohen argued that neither of these effects is under voluntary control, on the basis, in part, that the capture of attention at short intervals occurred even when the cue was not predictive of the target location.

Lambert, Spencer, and Mohindra (1987) examined this question further by providing explicit instructions to subjects to avoid attending to the cued location. They found that even under these instructions, subjects evidently could not completely avoid a tendency to attend to an abrupt peripheral cue. However, instructions did significantly reduce the tendency to attend to such cues, compared with a no-instruction condition. These results undermine the notion that attention is unavoidably and inevitably summoned by a peripheral cue.

In a recent study by Müller and Rabbitt (1989), subjects were to attend to a location cued by a central arrow; at various moments after the arrow appeared and before the target stimulus appeared, a box surrounding one of the four possible target positions brightened briefly. The box brightening was not predictive of target location and was to be ignored. Müller and Rabbitt found that performance was impaired when a box brightened at an uncued location, even when the cue correctly and predictably indicated the location of the target. They concluded that attention was involuntarily captured to some extent even when attention was directed to the cued location, although the extent of the performance impairment was modulated by voluntary allocation. It is worth noting that Müller and Rabbit used long cue-to-target stimulus onset asynchronies (SOAs; 600-1200 ms) and cues of moderate validity $(50 \%)$. Both of these factors might have prevented optimal attentional focusing, thus permitting some degree of attentional capture by the irrelevant box brightenings. In fact, in the experiments we report below, this is exactly what appears to happen.

In all of the studies we have reviewed in the preceding paragraphs, the effects of cues on capturing attention were examined. There was in all cases some period of time between the onset of the summoning event (the cue) and the imperative event (the target). In the experiments reported here, attention was allocated in advance, and the imperative event itself either did or did not have an abrupt onset. So the present approach may allow more direct assessment of the influence of abrupt peripheral onset on the capture of attention.

Another experiment that speaks to the intentionality criterion in a somewhat different domain was reported by Shiffrin and Schneider (1977, Experiment 4d). In this experiment subjects first underwent extensive training in a consistently mapped (CM) visual search task. CM training leads to automatic detection of target stimuli, as reflected in flat memoryand display-size functions. In order to demonstrate that $\mathrm{CM}$ targets also satisfy the intentionality criterion, Shiffrin and Schneider transferred these highly trained subjects to a new varied mapping (VM) visual search task in which none of the stimuli from the training task participated with the exception of occasional "CM foils" (i.e., letters that had been targets in the previous $\mathrm{CM}$ task). Subjects were to attend to one diagonal of a four-element display and to ignore the other diagonal. CM foils sometimes appeared in the to-be-ignored diagonal. The data revealed significant visual search interference on trials in which a CM foil was present compared with trials in which one was not, a result suggesting that subjects could not suppress an automatic attention response to the foil. Of course, attentional capture by a CM target is fundamentally different from attentional capture by an abrupt onset. The former is learned, whereas the latter may or may not be learned (e.g., it could be "hard-wired"). Nevertheless, this experiment provides further evidence that there may be conditions under which certain stimuli capture attention in violation of an individual's intentions.

In the experiments reported below, we pursued this question by testing the intentionality criterion for the automaticity of capture by abrupt-onset stimuli. According to this criterion, attention is said to be captured automatically only if capture cannot be prevented at will. In our earlier experiments (Yantis \& Jonides, 1984), subjects were not attempting to attend to any particular location; instead, they were prepared for the target in any position (in what Eriksen \& Yeh, 1985, called a diffuse attention mode). In the present experiments, in contrast, we provided subjects with advance information about the likely position of an upcoming target, to induce focused attention at a cued spatial location. We then examined the effects of an abruptly onset stimulus appearing in the cued or in an uncued location. If attention is captured automatically by an abrupt onset, reaction time to a target item should 
reflect capture whether attention was focused on it or not. On the other hand, if subjects are able to focus attention and prevent an abrupt onset from disrupting performance, the automaticity hypothesis must be reexamined.

\section{Experiment 1}

The purpose of Experiment 1 was to determine whether there is any reason to believe that abrupt onsets do not satisfy the intentionality criterion for automaticity. According to this criterion, the mere presence of an abrupt onset in a display should capture attention regardless of the subject's voluntary allocation of attention induced by an attentional precue. Consequently, whether the cue is valid or not should have little or no impact on performance in the presence of abrupt stimulus onset; in all cases the onset stimulus should exhibit priority.

On each trial of the task, a central cue appeared, indicating a position to the left or right of fixation. After $200 \mathrm{~ms}$, a letter appeared in each position. Subjects were to determine whether an $\mathrm{E}$ or an $\mathrm{H}$ appeared in the display (exactly one of these letters appeared in each display, with an irrelevant letter appearing in the opposite position). The cue was valid (i.e., indicated the position occupied by the target $\mathrm{E}$ or $\mathrm{H}$ ) on $80 \%$ of the trials. In this experiment we monitored eye position to ensure that covert movements of attention, and not overt movements of the eyes, were responsible for performance.

\section{Method}

Subjects. Seventeen University of Michigan undergraduates were paid to participate in two 50 -min sessions. All had uncorrected normal vision.

Equipment and stimuli. Stimulus events were controlled and responses were collected by a PDP-11/60 computer. Stimuli appeared on a DEC VT-11 graphics scope. Subjects responded by pressing keys on an HP-2621A terminal keyboard. Subjects sat in a sound-attenuating booth under comfortable illumination.

Eye position was monitored by a Gulf + Western Model 200 scleral reflectance device. Eye movement data were collected via an analogto-digital converter on the computer sampling at $1 \mathrm{kHz}$. Head position was maintained by a chin rest. The monitor was calibrated at the start of each block of trials and again whenever departures from fixation were detected on three trials since the last calibration run. A position criterion of $\pm 1.5^{\circ}$ horizontally was established for defining eye movements. Trials on which an eve movement was detected were marked for identification in subsequ at analyses; these trials were not rerun.

The stimulus letters were constructed by illuminating five of the seven segments of a box figure eight. The letters so constructed were E, H, P, S, and U. Each letter subtended a visual angle of $1.9^{\circ}$ in height and $1^{\circ}$ in width from a viewing distance of $45 \mathrm{~cm}$. The letters appeared $5.8^{\circ}$ to the left and to the right of fixation. The vertical separation of the nearest contours of the stimuli was $0.5^{\circ}$.

Two letter-presentation modes were used. Onset letters were presented by illuminating a five-segment letter in a location that had previously contained no segments. No-onset letters were presented by removing two segments from a seven-segment box figure eight to reveal a previously camouflaged five-segment letter. In the former case, the letter segments appeared abruptly; in the latter, the camouflaged letter segments were present for $1,200 \mathrm{~ms}$ before display onset and did not themselves change during the course of presentation. ${ }^{2}$
In order to equate the two presentation modes as much as possible, we began each trial with two placeholders on each side of the display. One of these was the required box figure eight, which would serve as camouflage for a no-onset letter (if one were to appear on that side of the display). The other placeholder consisted of six dots arranged at the vertices of a box figure eight. The two placeholders appeared one directly above the other, $0.5^{\circ}$ apart at their nearest contours, two on the left and two on the right of the display. They were illuminated $1,200 \mathrm{~ms}$ before the onset of the display. The relative positions (top or bottom) of the dot and figure eight placeholders were chosen randomly and independently for the two sides on each trial. At trial onset, one of the two placeholders on each side disappeared, and the other changed into a letter. On trials on which an onset letter appeared on a given side of the display, the figure eight placeholder disappeared, and the six dots changed into a letter. On trials on which a no-onset letter appeared on a given side of the display, the six-dot placeholder disappeared, and two segments of the figure eight were removed to reveal a letter. These events are illustrated in Figure 1.

Design. A $2 \times 2 \times 2$ design was used, the factors of which were target type (onset/no-onset), distractor type (onset/no-onset), and cue validity (valid/invalid). Target type and distractor type were completely crossed factors, so that half of all trials involved onset targets and half no-onset targets; within each of these conditions, half of the distractors were onset and half no-onset. Thus $25 \%$ of the trials fell under each of the four combinations of target and distractor type.

On $80 \%$ of the trials, the cue arrow indicated the side that the target $(\mathrm{E}$ or $\mathrm{H})$ would occupy, and on the remaining $20 \%$ of the trials, the cue indicated the opposite side. The target appeared on each side about equally often. The target was $\mathrm{E}$ on half of the trials and $\mathrm{H}$ on the other half. Target location, target identity, distractor identity (the distractors came from among $P, S$, and $U$ ), and the relative positions of the letters (top or bottom) within a side were chosen randomly on each trial. The ordering of trial types determined by the three main factors was also random. Each session was divided into 10 blocks of 40 trials each, for a total of 400 trials per session. Session 1 was considered practice.

Procedure. Trial events are depicted in Figure 1. A fixation cross, along with the four placeholders, was first illuminated. After 1,000 $\mathrm{ms}$ a single arrow appeared at fixation for $200 \mathrm{~ms}$, pointing either to the left or the right. Subjects were told to maintain fixation on the center of the screen throughout the trial (the penalty for repeated failure being recalibration of the eye movement monitor, a mildly annoying event). Finally, the placeholders were replaced by two letters, one on the left and one on the right, as described above. One of the two letters was the target to be discriminated $(\mathrm{E}$ or $\mathrm{H})$, and the other was an irrelevant distractor $(P, S$, or $U)$. Subjects determined which of the two targets was present and responded by pressing the period key (.) with their right index finger if the $\mathrm{H}$ appeared and the slash key ( / ) with their right middle finger if the $\mathrm{E}$ appeared. The display was then erased, and the next trial started $500 \mathrm{~ms}$ later. Error feedback was provided via an auditory beep. The duration of the cuing arrow was chosen so as to generate the maximum attentional facilitation typically observed in experiments of this type (e.g., Remington \& Pierce, 1984).

At the end of each block of trials, a performance summary giving mean reaction time and total number of errors was displayed. Subjects were encouraged to respond quickly while maintaining a low error rate (i.e., no more than one or two errors in each block of 40 trials).

\footnotetext{
${ }^{2}$ Abrupt offset of the camouflaging contours was shown by Yantis and Jonides (1984, Experiment 3) to have no significant disruptive effect on attentional capture by abrupt onset; however, see Miller (1989).
} 
Subjects were told that the target would be $\mathrm{E}$ half of the time and $H$ half of the time and that it would appear on the left and the right sides equally often. They were further told that the cuing arrow would provide important preparatory information: The target would appear on the side indicated by the arrow $80 \%$ of the time. It was made clear that it would be to their advantage to attend to the indicated side while maintaining fixation, so as to increase their speed and accuracy. Subjects readily understood and indicated compliance with these instructions.

\section{Results}

The results of Experiment 1 revealed that abrupt onset alone did not determine the focus of attention. Instead, whether the target was cued or not modulated the effect of abrupt onset. This conclusion is reflected in the results of a three-way analysis of variance (ANOVA), with target type (onset/no-onset), distractor type (onset/no-onset), and cue validity (valid/invalid) as factors. There was a significant main effect of validity, $F(1,16)=55.2, p<.001$, and target type, $F(1,16)=20.6, p<.001$; the effect of distractor type was not significant, $F(1,16)<1$. Most important, there was a significant interaction between cue validity and target type, $F(1$, 16) $=9.5, p<.01$; the effect of target type was larger when the cue was invalid $(25 \pm 12 \mathrm{~ms})$ than when it was valid $(8 \pm$ $9 \mathrm{~ms}$ ). ${ }^{3}$ This suggests that when subjects attended to the target position before its appearance, it mattered little whether the target had an abrupt onset but when subjects were misled by the cue, a target with an abrupt onset was more rapidly identified than one without an abrupt onset.

The comparison afforded by the factorial analysis above is not completely illuminating, however, because it includes the relatively uninformative conditions in which the target and disiractor were either both onset or both no-onset stimuli. It is more revealing to analyze separately the subset of the data in which the target was an onset and the distractor a no-onset, or vice versa (i.e., those trials on which exactly one onset and one no-onset stimulus appeared). An analysis of variance was therefore conducted with this subset of the data (shown in Figure 2).

There was a significant main effect of cue validity, $F(1,16)$ $=79.3, p<.001$ : Reaction time was much faster with a valid than an invalid cue. There was also a significant effect of

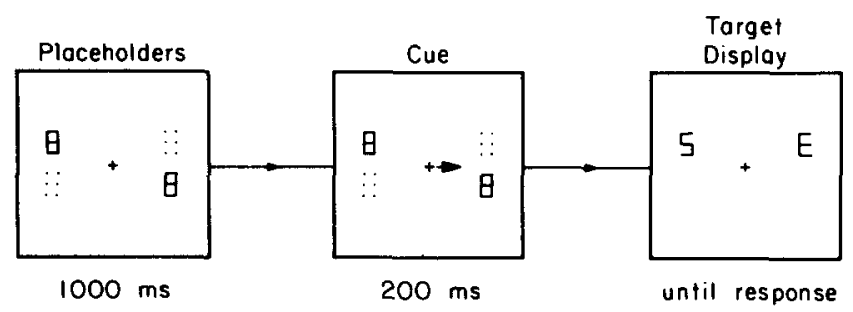

Figure 1. Trial events in Experiment 1. (In this example, the target is an $\mathrm{E}$ and the cue is valid; the target is an onset stimulus, and the distractor [S] is a no-onset stimulus. The relative positions of the figure eight and dot placeholders were varied from trial to trial with the constraint that there was always one of each on each side of fixation. Letters could appear in either the upper or the lower position on each side. The target could be either an $\mathrm{E}$ or an $\mathrm{H}$.)

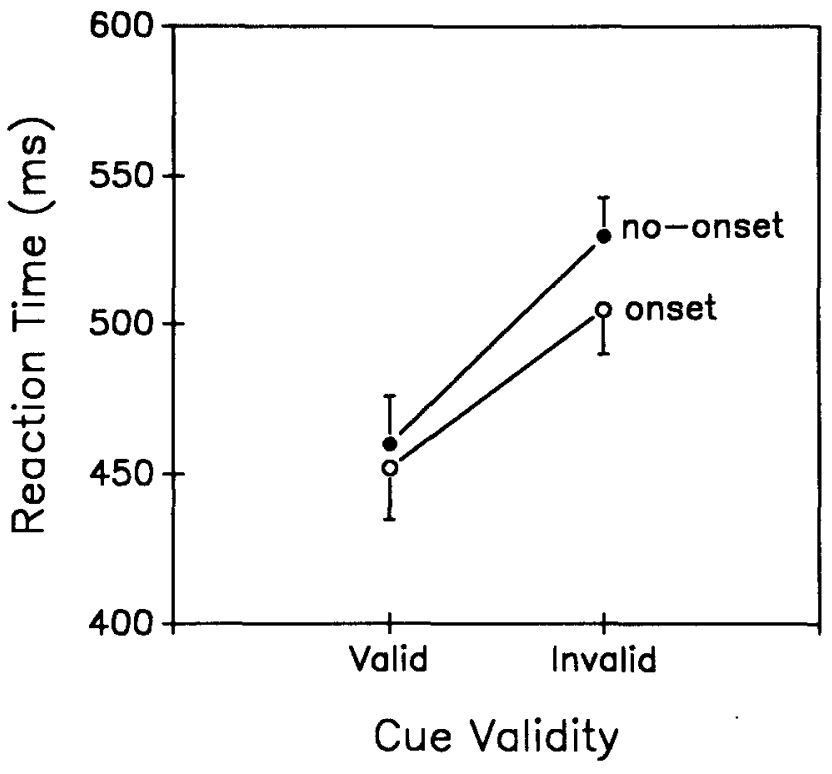

Figure 2. Mean reaction time for the conditions of Experiment 1 that involved one onset and one no-onset stimulus. (Curve parameter is target type. Error bars extend to $\pm 1 S E$.)

target type, $F(1,16)=32.5, p<.001$ : When the target was an onset and the distractor was not, reaction time was faster than when the distractor was an onset and the target was not. Finally, the interaction between these two factors was significant, $F(1,16)=8.2, p<.05$. The effect of target type was larger when the cue was invalid than when it was valid. This interaction is what calls into question the hypothesis that abrupt onset always captures attention involuntarily.

Error rate in this experiment was $5.3 \%$ overall. There was a strong positive correlation between error rate and mean reaction time (.64), which indicates that there was no strong speed/accuracy trade-off.

\section{Discussion}

The results of Experiment 1 provide preliminary evidence that abrupt onsets may not satisfy the intentionality criterion for strong automaticity. This conclusion would undermine our earlier speculation (Yantis \& Jonides, 1984) that abrupt onsets capture attention automatically.

To clarify this point, we considered what the very strongest version of the intentionality hypothesis would state. If abrupt onset captured attention regardless of the volitional state of the subject (e.g., whether or not the subject was focusing attention on a specific location in space), then reaction time to identify a target with an abrupt onset should not vary at

${ }^{3}$ Effect sizes are given throughout this article in terms of the mean $\pm S E$, rounded to the nearest millisecond. Because all within-subjects comparisons in Experiments 2 and 3 involved groups of 10 subjects, there are 9 degrees of freedom for $t$ tests using these values. Thus, whenever $\mathrm{M} / S E>2.262$, the effect is significant at the .05 level. Reported $t$ values may vary from the ratios of the values given in the text because of rounding. 
all with cue validity, and reaction time to onset targets should be faster everywhere than reaction time to no-onset targets. In other words, according to this account, the two functions in Figure 2 should be horizontal and parallel to one another, with the onset function below the no-onset function. It is clear that this pattern did not emerge.

A slightly weaker version of the hypothesis would state that there might be a small delay associated with drawing attention from an attended location to an unattended onset stimulus, which would yield an effect of cue validity, but that subjects would always take the time to process the onset stimulus. According to this account, reaction time in the valid-cue condition should have been influenced a great deal by whether the target was an onset or not: On trials when the target was an onset, reaction time should have been fast; on trials when the distractor was an onset, subjects' attention should have been captured by the distractor in the uncued position, which would result in a substantial delay in responding.

Indeed, the effect of target type in the valid-cue condition should have been at least as large as in the invalid-cue condition; in the invalid-cue condition, subjects would either be captured by the (uncued) onset target, which would yield a fast reaction time, or be required to process the (cued) onset distractor and then shift attention to the (uncued) target position. In other words, the effect of target type on the validcue trials should have been to add two shifts of attention and an analysis of a distractor to reaction time; the corresponding effect on the invalid-cue trials should have involved only one shift of attention and an analysis of the distractor. However, the data reveal that the effect of target type was significantly smaller on the valid- than on the invalid-cue trials.

Together, these detailed predictions of various versions of the intentionality criterion are clearly violated by the results from Experiment 1 and lead us to question whether abrupt onsets satisfy that criterion for automaticity.

The next task was to explore the boundaries of this violation of the intentionality criterion. In Experiments 2 and 3, respectively, we manipulated two factors that are thought to influence the allocation of attention: cue-target SOA and cue validity. This permitted us to determine whether the voluntary allocation of attention dominates capture by abrupt onset and, if so, to what extent.

In Experiments 2 and 3, we also removed the six-dot placeholders that were used in Experiment 1. The six-dot placeholders were used to address a confounding in previous experiments: Onset stimuli always appeared in blank locations, whereas no-onset stimuli appeared in nonblank locations. The purpose of the placeholders was to show that the unavoidable attentional capture induced by abrupt onsets could not be attributed to the absence of some kind of object in the location subsequently occupied by the onset stimuli. If there is some small effect of this manipulation, it would serve to diminish the influence of abrupt onset in Experiment 1. We found in Experiment 1 that abrupt onsets did not capture attention regardless of intention; this shifted our a priori null hypothesis to one stating that abrupt onsets do not capture attention involuntarily. Consequently, in Experiments 2 and 3 we did not use the six-dot placeholders in order to maximize the opportunity for attentional capture by abrupt onset.

\section{Experiment 2}

In Experiment 2, subjects were required to determine which of two letters ( $\mathrm{E}$ or $\mathrm{H}$ ) was present in a display of four letters arranged at a subset of the vertices of an imaginary hexagon centered at fixation. On each trial a central arrowhead cue reliably indicated the location of the critical letter. The cue appeared $200 \mathrm{~ms}$ before, simultaneous with, or $200 \mathrm{~ms}$ after the onset of the display. Subjects are known to be capable of aligning attention with a spatial location that is likely to contain task-relevant information within $200 \mathrm{~ms}$ of receiving that information (e.g., Eriksen \& St. James, 1986; Murphy \& Eriksen, 1987; Posner, 1980; Posner, Cohen, \& Rafal, 1982; Remington \& Pierce, 1984). Thus the leading cue placed subjects in a state of maximal attentional readiness for the appearance of the critical item, and the trailing cue did not permit subjects to align attention with the cued location before the display appears. The three cuing conditions were run with three separate groups of subjects. Crossed with the cuing factor was an onset factor. In each display, exactly one item had an abrupt onset, and the remaining three items did not have an abrupt cnset.

The automaticity hypothesis asserts that regardless of the subject's state of attentional readiness, an abrupt onset appearing in an uncued location should capture attention and slow responding to cued items that do not have abrupt onsets, because, by hypothesis, attention is involuntarily drawn to the onset location and can be applied only subsequently to the cued item. On the other hand, if the preliminary allocation of attention dominates performance, then whether or not the target has an abrupt onset should not influence performance.

\section{Method}

Subjects. Thirty undergraduates at the Johns Hopkins University served in one 50-min session for class credit. All subjects had normal or corrected-to-normal vision. Ten subjects were randomly assigned to each of the three SOA conditions.

Stimuli and e.quipment. Stimuli were presented on a NEC Multisync monitor driven by a Color- 400 EGA card controlled by an IBM PC/AT microcomputer. Letters were high-contrast yellow against a black background. The letters $E, H, P, S$, and $U$ served as stimuli. The letters were formed by illuminating the appropriate segments of a seven-segment box figure eight. From a viewing distance of about $40 \mathrm{~cm}$, each letter subtended $0.9^{\circ}$ of visual angle in height and $0.5^{\circ}$ in width. The letters appeared at a subset of the vertices of an imaginary hexagon centered at fixation with radius $4.2^{\circ}$, and they were $4.2^{\circ}$ apart, center to center. The cue was a triangular arrowhead, appearing directly at fixation, pointing at one of the six potential stimulus locations. The arrowhead subtended about $0.6^{\circ}$ in length and $0.3^{\circ}$ in width at its base (see Figure 3 for an example of the display).

Responses were made by pressing with the right or left index finger one of two buttons mounted in an angled response box placed on the table in front of the subject.

Design. Each subject participated in eight 72-trial blocks. A $2 \times$ 2 factorial design was used within subjects. The two within-subjects factors were target identity ( $\mathrm{E}$ or $\mathrm{H}$ ) and trial type (onset or no-onset). The target was an $\mathrm{E}$ on half of the trials in each block and an $\mathrm{H}$ on the other half. Within each of these factors, the target had an abrupt onset on half of the trials, and the three nontargets were of the noonset type; on the other half of the trials, one of the nontargets had 


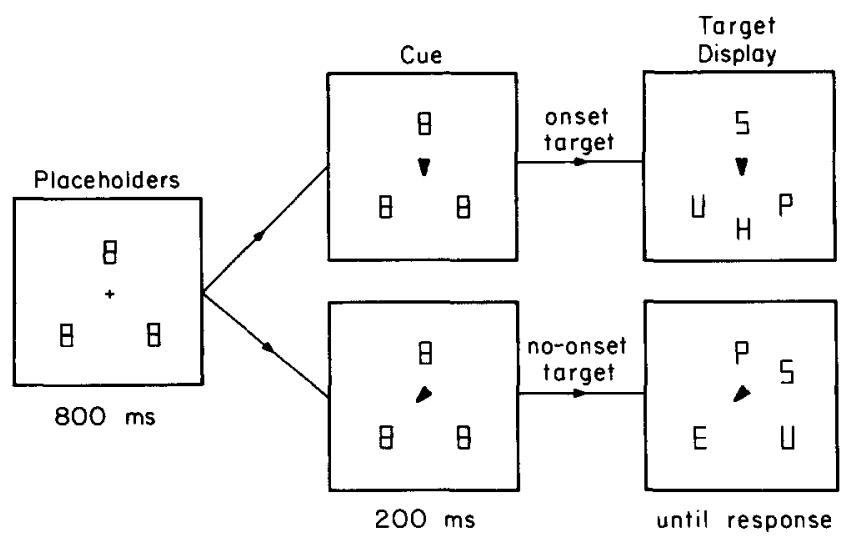

Figure 3. Trial events in the $-200-\mathrm{ms}$ stimulus onset asynchrony (SOA) condition of Experiment 2. (In the top row, right, the target $[\mathrm{H}]$ is an onset letter. In the bottom row, right, the target [E] is a noonset letter, and one of the distractors [S] is an onset. The cue in both examples precedes the target display by $200 \mathrm{~ms}$; in two other conditions, not shown, the cue appears simultaneous with or $200 \mathrm{~ms}$ after the start of the target display. The initial positions of the three figureeight placeholders alternated between the upward-pointing triangle, shown, and an inverted triangle.)

an abrupt onset, and the target and two of the distractors were of the no-onset type.

There was one between-subjects factor, SOA. The asynchrony between the onsets of the letter display and the cue was $-200 \mathrm{~ms}$ (i.e., the cue preceded the onset of the display by $200 \mathrm{~ms}$ ), $0 \mathrm{~ms}$ (i.e., the cue and the display appeared simultaneously), or $200 \mathrm{~ms}$ (i.e., the cue followed the display by $200 \mathrm{~ms}$ ). The cue pointed at the critical letter $(E$ or $H)$ on every trial and thus represented a $100 \%$ valid attention cue.

The target appeared in each display location equally often. On noonset trials, the location of the onset nontarget was chosen from each of the nontarget locations equally often.

A number of precautions were taken to avoid transient practice effects. The session began with a block of 20 practice trials; each block began with 3 warm-up trials, and every error was followed by a randomly chosen recovery trial. Responses from the practice, warmup, and recovery trials were not recorded and did not enter into any analyses.

Procedure. On each trial (see Figure 3) a fixation point was displayed for $500 \mathrm{~ms}$ as a warning that the trial was about to begin. Three box figure-eight placeholders were then displayed (along with the fixation point) at three of the stimulus positions, forming either an upward-pointing or a downward-pointing triangle (randomly and equally often within each block). The placeholders were always present for $1,000 \mathrm{~ms}$ before the onset of the test display regardless of SOA. The test display consisted of four letters. Three of these (the no-onset letters) appeared in the locations previously occupied by the placeholders; they were revealed by removing the appropriate segments of the figure eight, thereby avoiding abrupt onset of the contours of the letter itself. The fourth letter (the onset stimulus) appeared abruptly in a previously blank location at the same time that the no-onset letters were revealed.

The cue appeared at fixation and always indicated the location of the critical letter $(\mathrm{E}$ or $\mathrm{H})$. The cue appeared at one of three possible SOAs with respect to the start of the letter display: $-200,0$, or 200 ms. Because the placeholders were always present for $1,000 \mathrm{~ms}$ before the onset of the letters, the cue appeared $800,1,000$, or $1,200 \mathrm{~ms}$ after the onset of the placeholders.
Subjects were instructed to determine whether an $\mathrm{E}$ or an $\mathrm{H}$ was present in each test display and to press the right button with their right index finger if the $\mathrm{E}$ was present and the left button with their left index finger if the $\mathrm{H}$ was present. They were told to use the cue to direct their attention to the critical location, if possible. To reiterate, the cue was $100 \%$ valid: It always indicated the location of the critical letter. A $100-\mathrm{ms}, 800-\mathrm{Hz}$ tone was presented as feedback if an incorrect response was made. No tone was provided after correct responses.

Subjects were instructed not to move their eyes at any time during the experiment, as this would make their task more difficult and slow their responses. None of the subjects expressed any difficulty in maintaining steady fixation. Previous work in similar paradigms has shown that subjects can and do reliably maintain fixation when this is required; task replications under eye movement monitoring have repeatedly failed to detect any effect on performance. The results of Experiment 1, in which we did monitor eye position, support our contention that eye position remained constant in this experiment.

\section{Results}

Mean reaction times from correct responses for the various conditions of Experiment 2 are shown in Figure 4. The results are straightforward: Under an effective attentional cue (i.e., in the $-200-m s$ SOA condition), there was no reliable difference in reaction time between trials on which the critical item had an abrupt onset and trials on which one of the distractors had an abrupt onset. In contrast, when the cue was relatively ineffective (i.e., in the $0-\mathrm{ms}$ and $200-\mathrm{ms}$ SOA conditions), whether the target or a distractor had an abrupt onset had a large and significant effect.

To verify these trends, we carried out a two-way repeated measures ANOVA, with the between-subjects factor of SOA $(-200,0$, and $200 \mathrm{~ms})$ and the within-subjects factor of trial type (onset and no-onset). This analysis revealed a significant main effect of both factors, $F(2,18)=4.57, p<.05$ for SOA and $F(1,9)=39.60, p<.001$ for trial type. The interaction between SOA and trial type was also highly significant, $F(2$, 18) $=30.69, p<.001$. The effect of trial type was $8 \pm 5,52$ \pm 10 , and $117 \pm 18 \mathrm{~ms}$ for the $-200-, 0$-, and 200-ms SOA groups, respectively (see Footnote 3 ). Planned comparisons of these values revealed that the difference between the onset

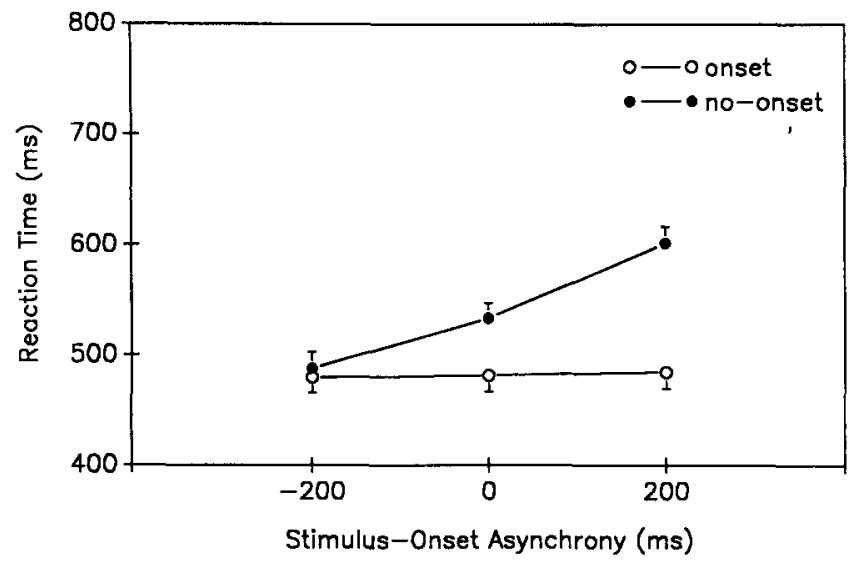

Figure 4. Mean reaction time for each condition of Experiment 2. (The bars extend to $\pm 1 S E$.) 
and the no-onset trials in the $-200-\mathrm{ms}$ group was not significantly greater than zero, $t(9)=1.71, p>.1$ and that the corresponding differences in the 0 - and 200-ms groups were significantly greater than zero, $t(9)=4.98$ and 6.45 for the two groups, respectively, both $p s<.001$.

The overall error rate was $2.4 \%$. Analysis of the error rate differences in the three SOA conditions revealed a pattern similar to that for the reaction times. The differences in error rate between the onset and the no-onset trials were $0.6 \pm 0.2$, $1.4 \pm 0.5$, and $2.6 \pm 0.9 \%$ for the $-200-, 0-$, and $200-\mathrm{ms}$ SOAs, respectively. There was a positive correlation of .93 between mean reaction time and mean error rate in the six experimental conditions. No suggestion of a speed/accuracy trade-off is present.

\section{Discussion}

A straightforward characterization of these data is that focusing attention in response to a valid and temporally useful cue (in the -200-ms SOA condition) virtually eliminated any effect of abrupt onset in the discrimination task. This can be seen by noting that the results for onset and no-onset targets are virtually identical in this condition. It should be recalled that when the target is of the no-onset type, there is an onset elsewhere in the display. If this distractor onset had captured attention, it should have disrupted performance on these trials compared with onset-target trials. It did not. Evidently, then, abrupt onset did not overcome the volitional allocation of attention in this experiment, and the intentionality criterion for automaticity was violated.

When the attentional cue was not available in advance of the onset of the test display in the $0-\mathrm{ms}$ and $200-\mathrm{ms}$ SOA conditions, attentional resources could not be focused in anticipation of the critical item. Under these circumstances, abrupt onset had a substantial influence on reaction time: When the target had an abrupt onset, responses were much more rapid than when one of the distractors had an abrupt onset. It is important to establish this result, because it demonstrates that abrupt onset can capture attention in the discrimination task used here, thereby providing validation of the task.

One might wonder why the effect of abrupt onset was greater in the 200 -ms condition than in the 0 -ms condition. After all, in both of these conditions, subjects were presumably unable to focus attention on the relevant position before the onset of the display. We suggest that attention was drawn to the onset position rapidly in both of these conditions (because in neither of them was attention focused in advance). Indeed, the data from the onset conditions suggest that attentional capture by abrupt onset was nearly as efficient in these conditions as when attention was allocated in advance. Now consider the no-onset conditions. Here, attention is assumed to be drawn to a distractor with an abrupt onset. In the 0-ms condition, the information from the cue might still have been used to shift attention from the distractor position to the cued target position. In the $200-\mathrm{ms}$ condition, however, information provided by the cue about the position of the target was not available until later, and so subjects may have had to engage in a search for the target until it was found or until the cue appeared. Because some search time may have been required in the $200-\mathrm{ms}$ no-onset condition, reaction time was slower than in the $0-\mathrm{ms}$ no-onset condition.

The finding that abrupt onsets capture attention when attention is unfocused is consistent with the results of Yantis and Jonides (1984). In that study, subjects were in a state of diffuse attention (there were no additional cues), and therefore they had not allocated attention to specific locations in preparation for the display. As in the present experiment, onsets did capture attention in that case.

The present experiment also verifies a conclusion drawn from Experiment 2 of Yantis and Jonides (1984). That experiment was conducted to determine whether the superior performance for onset stimuli over no-onset stimuli might be attributed not to attentional capture but to some perceptual difficulty in processing no-onset stimuli compared with onset stimuli. In the experiment subjects were provided with an advance cue indicating the position of a target that was to be identified. We found that reaction time to discriminate the target was the same for onset and no-onset stimuli, as long as attention was suitably allocated in advance. This demonstrated that when differential attentional effects were removed, there was no residual difference in the time required to process onset and no-onset letters. The present experiment verifies and extends that result: When attention is effectively allocated in advance, reaction time to discriminate a letter does not depend on whether it is an abrupt onset. The present experiment goes further in demonstrating that this holds even when a to-be-ignored onset stimulus appears elsewhere in the visual field.

\section{Experiment 3}

Experiment 2 established that an effective attentional cue could subvert the attention-capturing power of an abrupt onset. In that experiment we manipulated the effectiveness of the attentional cue by varying its temporal position. Less effective cues (i.e., those that could not be used to focus attention in advance of the display) yielded performance that was most susceptible to capture by abrupt onset. To provide converging evidence for this conclusion, we conducted another experiment in which we again manipulated cue effectiveness, this time by varying the predictive validity of the cue while keeping its temporal position fixed at $-200-m s$ SOA (which has been shown to be effective in focusing attention, as verified by Experiment 2; see also Eriksen \& St. James, 1986; Remington \& Pierce, 1984). The cues had validities of $100 \%, 75 \%$, or $25 \%$ (with four stimulus positions, $25 \%$ provides only random predictive validity).

The results of Experiments 1 and 2 already undermine a strong version of the automatic-capture hypothesis. On the basis of those results, we hypothesized that functional (i.e., high-validity) cues would tend to rivet attention to the cued position and thus prevent the disruption of performance by an onset occurring elsewhere in the field. It should be noted that we obtained just this result in the -200 -ms SOA condition of Experiment 2. In contrast, when the cue was not as functional (i.e., in low-validity conditions), subjects would be likely to spread their attention over the entire display area, 
and the appearance of an onset should capture attention and dominate performance. This hypothesis, then, predicts an interaction between cue validity and trial type.

\section{Method}

Subjects. Thirty undergraduate students at the Johns Hopkins University served as subjects for course credit. None of the subjects had served in Experiments 1 or 2, and all had normal or correctedto-normal vision. Ten subjects were randomly assigned to each of three validity groups.

Stimuli and equipment. The stimuli and equipment were the same as in Experiment 2.

Design. Each subject participated in eight blocks of 72 trials. The two within-subjects factors in the $2 \times 2$ design were target identity ( $E$ or $\mathrm{H}$ ) and trial type (onset or no-onset). As before, the target was an $E$ on half of the trials in each block and an $\mathrm{H}$ on the other half; within each of these factors, the target had an abrupt onset on half of the trials (and the three nontargets were of the no-onset type), and on the other half of the trials one of the nontargets had an abrupt onset (and the target was of the no-onset type).

The between-subjects factor was cue validity. One group of subjects experienced a cue validity of $100 \%$, one group $75 \%$, and one group $25 \%$. For the $75 \%$ group, the cue indicated the location of the target on $75 \%$ of the trials and a randomly chosen nontarget location on the remaining trials. For the $25 \%$ group, the cue indicated the target on just $25 \%$ of the trials and a randomly chosen nontarget on the remaining $75 \%$ of the trials. This yields five conditions, which we denote as follows: $100 \mathrm{~V}, 75 \mathrm{~V}, 75 \mathrm{I}, 25 \mathrm{~V}$, and $25 \mathrm{I}$. The number corresponds to the validity condition $(100 \%, 75 \%$, or $25 \%$ validity), and the letter corresponds to whether the cue was valid or invalid (V or I). Thus, Condition $75 \mathrm{I}$ consisted of those $25 \%$ of the trials for the $75 \%$ validity group on which the cue was not valid. Similarly, Condition 25 I consisted of those $75 \%$ of the trials for the $25 \%$ validity group on which the cue was not valid. Of course, there were no invalid trials for the $100 \%$ validity group.

Each condition can be categorized according to whether the target had an abrupt onset. The target appeared in each location equally often. On no-onset trials the location of the onset nontarget was chosen from each of the nontarget locations equally often.

The 100\%-valid group underwent exactly the same stimulus conditions as the $-200-\mathrm{ms}$ SOA group in Experiment 2 (all SOAs in the present experiment were $-200 \mathrm{~ms}$, and all cue validities in Experiment 2 were $100 \%$ ). This condition therefore represents an exact replication of the corresponding condition of Experiment 2.

As in Experiment 2, we included practice, warm-up, and recovery trials in the design, the responses to which were not recorded and did not enter into any analyses.

Procedure. The procedure was just as in Experiment 2 with the exception that the cue always appeared $200 \mathrm{~ms}$ before the onset of the test display and that the cue sometimes indicated a nontarget location (for the $75 \%$ and the $25 \%$ groups). Figure 3 shows the sequence of stimulus events.

Subjects in all three groups were informed of the effective cue validity. Subjects in the $100 \%$ and in the $75 \%$ conditions were encouraged to attend to the cued location, because that location was certain (for the $100 \%$ group) or likely (for the $75 \%$ group) to contain the target item on each trial. Subjects in the $25 \%$ condition were told that the target was equally likely to appear in any of the display locations (including the cued one) regardless of where the cue pointed. They were told to ignore the cue and to be prepared to make a perceptual judgment about stimuli in any of the display locations. We thus attempted to encourage subjects in the $25 \%$ group to remain in a diffuse attention mode.

\section{Results}

Mean reaction times from correct responses for the various conditions of Experiment 3 are shown in Figure 5. There was no effect of target type in the $100 \mathrm{~V}$ condition. This finding, which verifies the result obtained from the -200 -ms SOA condition of Experiment 2, demonstrates that highly focused attention can overcome attentional capture by abrupt onset. In the $75 \%$ and $25 \%$ conditions, in contrast, there was a larger effect of abrupt onset even on valid trials and a very large effect of abrupt onset on invalid trials.

A two-way ANOVA, with the between-subjects factor of cue validity $(100 \mathrm{~V}, 75 \mathrm{~V}, 75 \mathrm{I}, 25 \mathrm{~V}$, and $25 \mathrm{I})$ and the withinsubjects factor of trial type (onset or no-onset), revealed a significant main effect of both factors, $F(4,36)=27.01$ for validity and $F(1,9)=85.23$ for trial type, both $p s<.001$. The interaction between validity and trial type was also significant, $F(4,36)=13.46, p<.001$. A clearer picture of the data is obtained by examining the individual effects of abrupt onset for each validity condition.

The effect of trial type was $17 \pm 10,48 \pm 6$, and $46 \pm 11$ $\mathrm{ms}$ for the $100 \mathrm{~V}, 75 \mathrm{~V}$, and $25 \mathrm{~V}$ conditions, respectively (see Footnote 3). The effect of trial type for the 75I and 25I conditions was $121 \pm 9$ and $69 \pm 19$, respectively. Individual $t$ tests of these effects revealed that the difference between the onset and the no-onset trials in the $100 \mathrm{~V}$ condition was not significantly greater than zero, $t(9)=1.8, p>.1$, and that the corresponding differences in the $75 \mathrm{~V}$ and $25 \mathrm{~V}$ groups were significantly greater than zero, $t(9)=8.4$ and 4.3 for the two groups, respectively, both $p \mathrm{~s}<.01$. The effects in the two invalid conditions were also reliable, $t(9)=13.9$ and 3.6 for the $75 \mathrm{I}$ and $25 \mathrm{I}$ conditions, respectively.

The overall error rate was $3.0 \%$. There was a positive correlation of .90 between mean reaction time and mean error rate in the 10 experimental conditions, a result suggesting that there was not a speed/accuracy trade-off.

\section{Discussion}

We consider first the $100 \mathrm{~V}$ condition. As in the corresponding condition of Experiment $2(-200-\mathrm{SOA})$, there was vir-

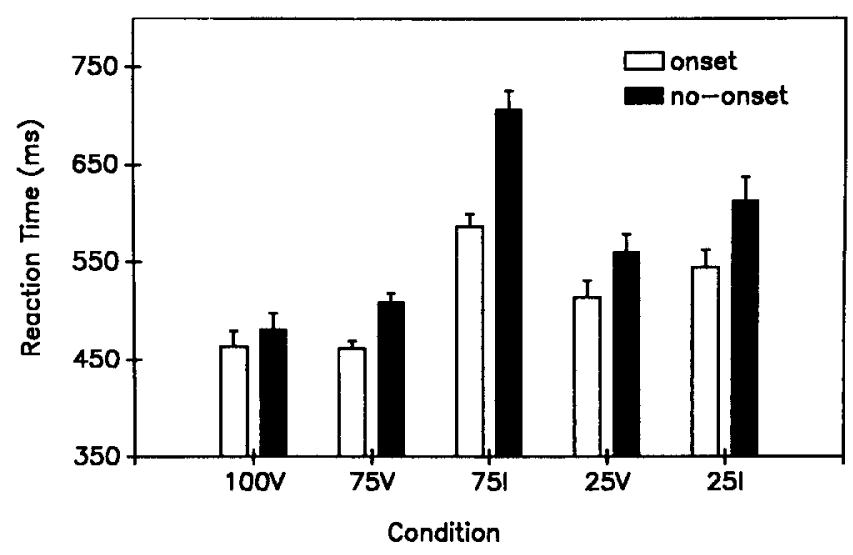

Figure 5. Mean reaction time for each condition of Experiment 3. (The error bars represent $1 S E$.) 
tually no effect of target type. This replicates our finding that when attention is effectively focused on a spatial location, abrupt onset does not capture attention.

Next we look at the $25 \%$ condition ( $25 \mathrm{~V}$ and $25 \mathrm{I}$ in Figure 5). If subjects had completely ignored the cue (as they should have, given its low validity and our instructions), we would expect the bars representing the $25 \mathrm{~V}$ and the $25 \mathrm{I}$ conditions to be the same height, to reflect the irrelevance of the cue. The bars are clearly not the same height. However, neither are they very different (compared, e.g., with the corresponding bars in the $75 \%$ validity conditions). To the extent that subjects were in something like a diffuse attention mode here, we would expect to see a noticeable effect of trial type (onset vs. no-onset), according to our hypothesis. This prediction was satisfied: Onset targets were identified more rapidly than no-onset targets were (an average difference of $57 \mathrm{~ms}$ ).

We now discuss the $75 \%$ condition ( $75 \mathrm{~V}$ and $75 \mathrm{I}$ in Figure 5). There are several possible strategies that a subject could adopt when the cue is highly, but not completely, valid. We consider three of the most plausible here. The first (Strategy 1) is always to attend maximally to the cued location, even though this is known to be harmful on some trials. This strategy is disconfirmed by the data, however, because it predicts that in the $75 \mathrm{~V}$ condition, the effect of target type should be as small as it is in the $100 \mathrm{~V}$ condition, and this is clearly not the case. The mean differences between the onset and the no-onset conditions were $17 \pm 10 \mathrm{~ms}$ for the $100 \mathrm{~V}$ condition and $48 \pm 6 \mathrm{~ms}$ for the $75 \mathrm{~V}$ condition, a significant difference, $t(18)=2.3, p<.05$. Evidently, then, Strategy 1 was not used by subjects in this task.

The second strategy for the $75 \%$ condition (Strategy 2 ) is to probability match over trials (Jonides, 1980), attending to the cued location on a randomly selected $75 \%$ of the trials and either selecting a random uncued position to attend to on the remaining trials (Substrategy 2A) or remaining in the diffuse attention state on those trials (Substrategy 2B; Eriksen \& Yeh, 1985).

These strategies predict a probability mixture of two different attentional states on valid trials: those on which attention is highly focused (as in the $100 \mathrm{~V}$ condition) and those on which attention is either focused incorrectly (Substrategy 2A) or unfocused (Substrategy 2B). In other words, Strategy 2 predicts that the distribution of reaction times in the $75 \mathrm{~V}$ onset and no-onset conditions should be a 75/25 mixture of the corresponding distributions in the $100 \mathrm{~V}$ and the $75 \mathrm{I}$ (Substrategy 2A) or the $100 \mathrm{~V}$ and $25 \mathrm{~V}$ (Substrategy 2B) conditions. ${ }^{4}$ The power required for formal statistical tests of this prediction is not sufficient for these data (see Meyer, Yantis, Osman, \& Smith; 1985; Smith, Yantis, \& Meyer, 1990, for details). Qualitatively, however, the data do not strongly violate the predictions of Strategy 2 .

The third strategy (Strategy 3) is to devote on each trial $75 \%$ of one's attentional resources to the cued location and $25 \%$ to the uncued locations (Shaw, 1978). This strategy predicts that in Condition $75 \mathrm{~V}$ there should be an effect of target type (onset vs. no-onset) that is larger than when attention is completely focused (Condition 100V) but less than when attention is diffuse (Condition 25V). This derives from the idea that attention is not completely focused in the
$75 \mathrm{~V}$ condition and so may be subject to partial capture, but it is not completely diffuse and so should be resistant to complete capture. ${ }^{5}$ An idea like this one was also proposed by Müller and Rabbitt (1989). The results confirm one aspect of this model: The effect of onset versus no onset is larger in the $75 \mathrm{~V}$ than in the $100 \mathrm{~V}$ condition. However, the effect of onset in the $75 \mathrm{~V}$ condition is not smaller than in the $25 \mathrm{~V}$ condition ( $48 \pm 6$ vs. $46 \pm 11$, respectively).

A further prediction of Strategy 3 is that both overall reaction time and the effect of target type should be much larger in the $75 \mathrm{I}$ condition than in the $75 \mathrm{~V}$ condition. This prediction is supported by the significant main effect of validity ( $75 \mathrm{~V}$ vs. $75 \mathrm{I}), t(9)=13.6, p<.001$, and the significant interaction between target type (onset vs. no-onset) and validity $(75 \mathrm{~V}$ vs. $75 \mathrm{I}), t(9)=11.7, p<.001$.

We conclude that the data are consistent with the predictions of Strategy 2 or Strategy 3. They are not consistent, however, with the hypothesis that abrupt visual onset captures attention automatically regardless of attentional state. Experiment 3 has demonstrated that the specific attentional strategy adopted by a subject given partially valid cues significantly influences the extent to which abrupt onset captures attention and verifies the conclusion from Experiments 1 and 2 that abrupt visual onset does not satisfy the intentionality criterion of strong automaticity.

The results of Experiment 3 also help to reconcile the present findings and those of Müller and Rabbit (1989). In their study, a to-be-ignored peripheral flash interrupted the detection of a target in a cued location. However, because the cue was only moderately valid $(50 \%)$, it cannot be said to have placed subjects into a state of highly focused attention. In the present experiment, even validities of as high as $75 \%$ yielded significant effects of to-be-ignored abrupt onsets. It is only when attention is highly focused in advance (as in the $100 \mathrm{~V}$ condition) that irrelevant abrupt onsets can be overridden by voluntary attentional focussing.

\section{Experiment 4}

We have ruled out versions of automatic attentional capture by abrupt onset in which attention is always immediately drawn to an abrupt onset regardless of subjects' intention. This is a strong version of automaticity that specifies a complete lack of control. However, there are less stringent (and perhaps more plausible) versions of automaticity that should

\footnotetext{
${ }^{4}$ The data suggest that subjects were not completely unfocused in the $25 \mathrm{~V}$ condition; instead, there is evidence that subjects used the cue to direct attention, even when it was randomly associated with the target location. To the extent that subjects were indeed using the cue in Condition $25 \mathrm{~V}$, it would not be appropriate to use data from that condition as one basis distribution in a test of the mixture prediction of Substrategy 2B.

${ }^{5}$ The idea implicit in this prediction is that because one can flexibly allocate portions of attention to different spatial locations, it follows that portions of attention can be captured by an abrupt onset; the more attention is allocated to a position, the less attention will be drawn away by an abrupt onset.
} 
still be entertained. It could be the case that an abrupt onset is always registered as an important, high-priority event by the visual system, but when attention is focused elsewhere, the "interrupt" generated by the onset is placed in a queue to be serviced only after attention is freed from its current task. In Experiment 4, we tested such a version of automaticity. ${ }^{6}$

In the experiment we used an interference paradigm (e.g., Eriksen \& Schultz, 1979; Yantis \& Johnston, 1990). On each trial of the task, a different letter was assigned to each of two responses. One position in the display was cued in advance, and the task was to respond according to the letter in the cued location. Uncued locations were usually occupied by neutral letters. On some trials, however, the letter assigned to the incorrect response for the trial appeared in one of the uncued positions. As before, the cued stimulus or one of the uncued stimuli could have an abrupt onset; the remaining letters were always no-onset letters.

With this paradigm, we can assess the extent to which interference from to-be-ignored items (if any) depends on whether those items have an abrupt onset. To perform this test, we must first establish that onsets per se do not interfere with the identification of the cued stimuli, in replication of our earlier results. Then we must determine whether there is any interference from to-be-ignored positions at all (indeed, it is possible to maximize the degree of interference through the careful choice of task parameters) and, if so, whether that interference is modulated by whether the interfering stimulus has an abrupt onset.

If interference is greater when the interfering letter is an onset rather than a no-onset item, the idea that the onset stimuli are registered as high priority by the visual system is supported. This would be consistent with the weaker view of automaticity described above. On the other hand, if the effect of the interfering item does not depend on whether it has an abrupt onset, the notion that onsets automatically capture attention, even in a weak sense, is undermined.

In this experiment we enhanced the potential for interference from to-be-ignored stimuli by varying the stimulus-toresponse mapping on each trial. This kind of procedure causes the mappings to be highly primed and may permit them to penetrate the filter established when attention is focused.

\section{Method}

Subjects. Sixteen undergraduates from the Johns Hopkins University ( 9 female) served in one 50 -min session to satisfy a requirement for an introductory psychology course. All subjects had normal or corrected-to-normal vision, and none had participated in Experiments $1-3$.

Equipment and stimuli. The equipment and stimuli were the same as in Experiment 2.

Procedure. At the start of each trial, two target letters appeared on the screen for $500 \mathrm{~ms}$, one above the right response key and one above the left response key. This was followed by a display consisting of three placeholders and a fixation cross for $1,000 \mathrm{~ms}$. A cue as in Experiment 3 then appeared for $200 \mathrm{~ms}$. Of course, this cue was always $100 \%$ valid, because the task was defined such that the cued letter was the one to be reported. Finally, the display of four letters appeared; one of these had an abrupt onset, and the other three were no-onset letters. One of the two target letters always appeared in the cued location, and subjects were to press the key corresponding to the cued target letter. The display disappeared when the response was made, and a beep was emitted if the response was in error. The next trial started after $1,000 \mathrm{~ms}$.

Design. The left and right responses were each required on half of the trials. The cued target letter was an onset letter on half of the trials, and one of the uncued letters was the onset on the remaining trials. On half of the trials of each of these types, there was an interfering letter (i.e., the target letter mapped onto the incorrect response) present in an uncued position, and on the remaining trials all the uncued positions were occupied by neutral letters. When the cued letter was a no-onset one and an interfering letter was present, the interfering letter was an onset on half of the trials and a no-onset on the other half.

There were eight blocks of 64 trials each. In each block, then, the cued target was an onset on 32 trials; an interfering target was present on 16 and absent on 16 of these trials. When the cued target was a no-onset ( 32 trials), the interfering target was again present on 16 trials and absent on 16 trials. Of the trials in which the target was a no-onset and the interfering target was present (16 trials), the interfering target was an onset on 8 trials and a no-onset on 8 trials. It is the results from this last pair of conditions that are the focus of this experiment.

\section{Results}

Mean reaction times for the main conditions of Experiment 4 are shown in Table 1. These data were subjected to a twoway analysis of variance, with cued target type (onset vs. noonset) and distractor type (interfering target present vs. absent) as factors. The 3-ms main effect of cued target type was not significant, $F(1,15)=1.2, p>.2$. The 33-ms main effect of the interfering target was highly significant, $F(1,15)=143.1$, $p<.001$. The interaction between these factors was not significant, $F(1,15)<1$. This analysis establishes that when subjects attended to the cued position, it mattered not at all whether the cued target was an abrupt onset or the onset was elsewhere in the display. This replicates the observed pattern of Experiments 1-3: abrupt onsets do not capture attention if attention is directed effectively in advance. The analysis also revealed that the identity of the to-be-ignored items did matter to some extent; that is to say, on those trials in which an interfering target appeared in an uncued location, reaction time was slowed compared with that on trials in which the interfering target did not appear. This result was obtained by design, of course: We selected task parameters that would maximize the possibility of interference from to-be-ignored letters.

What remains to be determined is whether the interference was modulated by whether the interfering target had an abrupt onset. For this analysis, we looked at the subset of trials on which the onset stimulus was one of the uncued letters and an interfering target was present. Reaction time when the interfering target was an onset was $525 \mathrm{~ms}$, and when it was a no-onset (and one of the other uncued letters was an onset), reaction time was $513 \mathrm{~ms}$. This effect of $12 \pm 4 \mathrm{~ms}$ was

\footnotetext{
${ }^{6}$ We thank Roger Remington for encouraging us to pursue this possibility. Preliminary evidence from a different paradigm used in our laboratory suggests that something like this model may be correct (Yantis \& Johnson, 1990).
} 
Table 1

Mean Reaction Time (in $\mathrm{ms}$ ) of Each Condition of Experiment 4

\begin{tabular}{cccc}
\hline \multirow{2}{*}{$\begin{array}{c}\text { Interfering } \\
\text { target }\end{array}$} & Onset & No onset & $M$ \\
\cline { 2 - 4 } & 514 & 519 & 516 \\
Present & 483 & 484 & 483 \\
Absent & & 501 & \\
$M$ & 498 & 5 target \\
\hline
\end{tabular}

significantly greater than zero, $t(15)=2.7, p<.02$. This effect demonstrates that even though attention was not immediately captured by the uncued abrupt onset, the interfering effect of a to-be-ignored stimulus was modulated by whether it had an abrupt onset. Thus the attentional effect of an abrupt onset evidently persists for some time beyond its immediate occurrence.

\section{Discussion}

According to the weaker version of automaticity we are testing, interfering targets that are themselves onsets should have a larger effect than those that are not onsets, even given that attention was not immediately drawn by the onset stimulus. The results of Experiment 4 are consistent with this model. Evidently, abrupt onsets may maintain the ability to summon attention, even if they are prevented from doing so immediately because attention is focused elsewhere.

The results we have described require that the effects of interfering stimuli in a task such as the one used in this experiment must be mediated by a series of overlapping processes. According to this account, attention is first allocated to the cued location, and information is extracted from that location; then attention goes on to the next-highest priority location, which in this case would be the onset location (if any). Information extracted from that location influences responses to the letter in the cued locationpresumably via a response-competition mechanism.

\section{General Discussion}

Four experiments were conducted to test the hypothesis that abrupt visual onset captures attention automatically. In Experiment 1, we showed that subjects' state of attentional readiness does modulate the extent to which abrupt onsets draw attention; this was essentially an existence proof. In Experiment 2, the effectiveness of a completely valid spatial cue was manipulated by presenting it before, simultaneous with, or after the appearance of a test display. Precues, which can, with the proper spatiotemporal properties, promote highly focused attention (e.g., Yantis \& Johnston, 1990), virtually eliminated the effect of abrupt onset on performance. In contrast, cues presented simultaneously with or after the onset of the display yielded strong effects of abrupt onset. We concluded that highly focused attention is resistant to attentional capture by abrupt onset.

In Experiment 3, we pursued this finding by manipulating the predictive validity of the cue. As in Experiment 2, with highly focused attention induced by completely valid cues, there was little effect of abrupt onset. However, with cues of lower validity, which evidently resulted in more diffuse attention allocation, abrupt onsets had a strong effect on performance.

In Experiment 4, we tested a somewhat weaker form of automaticity that might still be satisfied by abrupt onsets. We found that although abrupt onsets have no immediate effect on performance, they may still have a delayed or secondary effect in that they potentiate the interference caused by a response-incompatible letter in a to-be-ignored location. The picture emerging from this finding is that onsets may produce a "priority signal" that enters a queue. The signals in the queue are serviced in order of priority. When subjects are in a diffuse attention mode, an onset will have the highest priority, and attention will be captured. When attention is focused because of task demands, the focused location will have the highest priority and will be serviced before the nexthighest priority location, which might be the location containing an onset. Other preliminary evidence from our laboratory suggests that if there are multiple onsets present among a number of no-onsets, up to four of the onset stimuli may be serviced before any of the no-onsets are (Yantis \& Johnson, 1990). This is also consistent with the queuing notion.

One possible challenge to our conclusions is presented by our use of cues that themselves exhibit abrupt onsets. One might think that onset cues would interrupt the attentional capture produced by abrupt-onset stimuli and thereby compromise the effect of attentional capture by abrupt-onset letters with unwanted attentional capture by abrupt-onset cues. Upon closure scrutiny, however, it will be seen that the use of abrupt-onset cues is not problematic. First, the cues in these experiments are central ones. Jonides (1981) showed that central cues do not effectively capture attention (as peripheral cues, for example, do).

Second, one should consider the results of Experiment 3. In that experiment the cues always appeared $200 \mathrm{~ms}$ before the onset of the display. By the time the display itself appeared, attention was already effectively focused on the cued position, as indicated by the negligible effect of target type in the $100 \%$ validity condition. Consequently, we conclude that any possible attentional capture by the abrupt-onset cues had already dissipated by the time the display appeared $200 \mathrm{~ms}$ later.

Finally, we consider the results of Experiment 2. The condition that is most likely to present problems for our analysis is the 200 -ms SOA condition in which the cue appeared 200 $\mathrm{ms}$ after the onset of the display. These abrupt-onset targets were identified just as rapidly as in the -200 -ms condition, a result suggesting that the abrupt-onset targets did effectively capture attention; if the 200-ms cue had disrupted target processing, we would have expected reaction time in the 200ms onset condition to be slower than the -200 -ms onset condition. That it was not bolsters our claim that the abruptonset cue did not compromise the experiment. A similar argument applies to the 0-ms condition of Experiment 2.

Another feature of the data that merits comment is the effect of onset type in the -200 SOA, $100 \%$ valid conditions of Experiments 2, 3, and 4. Although in any given experiment this effect was not significantly greater than zero, it was 
consistently positive in all experiments. One might wonder if a real effect of onset was present but was too small to be statistically detected. We would argue that a more plausible explanation for this small but consistent effect is that on some small proportion of the trials, subjects may not have focused their attention with maximal efficiency (e.g., they were momentarily distracted or they lost concentration for a trial). On those trials we would expect that their attention would be drawn to the abrupt-onset stimulus. Indeed, it would be surprising if there were not at least some trials of this sort. This mixture of trials on which subjects focused well and onsets had no effect (most trials) and trials on which subjects did not focus well and onsets had a strong effect (a few trials) would be reflected in a small effect of onsets overall. However, because the effect of onsets was so small, the power of any formal statistical tests of this mixture hypothesis would not be sufficient to draw meaningful conclusions (Smith et al., 1990).

\section{Conclusion}

The overall conclusion to which we are led is that attentional capture by abrupt stimulus onset is not strongly automatic because, although it satisfies the load-insensitivity criterion, it does not strictly satisfy the intentionality criterion. The visual attention system is evidently prepared to give high priority to abrupt onsets when in diffuse attention mode. This is adaptively sensible, because onsets usually signal novel stimulus information, and this information may often be perceptually important. At the same time, the system delays the potentially distractive influence of abrupt onset when other task demands have led to highly focused attentionalso adaptively sensible. The mechanism for delaying the servicing of an abrupt onset may involve the setting of priorities that mandate the place where attentional resources must be committed.

The notion that priority signals are generated and queued to be serviced by the system as resources become available accounts for the results of Experiment 4 in which a to-beignored onset stimulus evidently had a higher priority than a to-be-ignored no-onset stimulus did. In addition, this account provides an explanation for the advantage of onsets over noonsets in the invalid conditions of Experiments 1 and $3 .^{7}$ In those conditions attention was focused on a position not containing a target letter; the effect of onset versus no-onset on target identification could have been mediated by a persisting code specifying higher priority for the onset than the no-onset items.

One possible computational mechanism that could support this kind of scheme was proposed by Koch and Ullman (1985). According to their model, visual information occupying the position in the visual field of highest salience or conspicuity is marked and passed on to a central representation that is responsible for further stimulus analysis. The priority code generated by an onset detector could easily be incorporated into this scheme and serve to activate nodes in Koch and Ullman's "saliency map" at the position of an abrupt onset. The even higher priority associated with a cued location could be represented on the saliency map as well.
The conclusion we have advanced is that attentional capture by abrupt visual onset does not satisfy all the common criteria for strong automaticity (Kahneman \& Treisman, 1984); therefore it should not, perhaps, be termed strongly automatic. A quite different conclusion is available, however. It is possible that the intentionality criterion for automaticity is not adequately diagnostic and should be abandoned or reformed (see Jonides et al., 1985). Of course, this issue has no objective answer. Definitions are not subject to empirical scrutiny. The criteria we choose to apply in ascribing automaticity to a cognitive or perceptual process are valid precisely to the extent that they are theoretically useful. If there are few processes that can satisfy the intentionality criterion, or if there are processes that appear prima facie to be good examples of automatic processes and yet do not satisfy the intentionality criterion, then the criterion itself may be suspect. The present data concerning abrupt onset represent one example of this type, which, if corroborated by other converging sources of evidence, may undermine the utility of the intentionality criterion.

\footnotetext{
${ }^{7}$ We thank Jeff Miller for pointing this out.
}

\section{References}

Breitmeyer, B. G., \& Ganz, L. (1976). Implications of sustained and transient channels for theories of visual pattern masking, saccadic suppression, and information processing. Psychological Review, 83 , $1-36$.

Cleland, B. G., Levick, W. R., \& Sanderson, K. J. (1973). Properties of sustained and transient cells in the cat retina. Journal of Physiology, 228, 649-680.

Egeth, H., Jonides, J., \& Wall, S. (1972). Parallel processing of multielement displays. Cognitive Psychology, 3, 674-698.

Eriksen, C. W., \& St. James, J. D. (1986). Visual attention within and around the field of focal attention: $\mathrm{A}$ zoom lens model. Perception \& Psychophysics, 40, 225-240.

Eriksen, C. W., \& Schultz, D. W. (1979). Information processing in visual search: A continuous flow conception and experimental results. Perception \& Psychophysics, 25, 249-263.

Eriksen, C. W., \& Yeh, Y. Y. (1985). Allocation of attention in the visual field. Journal of Experimental Psychology: Human Perception and Performance, 11, 583-597.

Hasher, L., \& Zacks, R. T. (1979). Automatic and effortful processes in memory. Journal of Experimental Psychology: General, 108, 356-388.

Jonides, J. (1980). Towards a model of the mind's eye's movement. Canadian Journal of Psychology, 34, 103-112.

Jonides, J. (1981). Voluntary vs. automatic control over the mind's eye's movement. In J. B. Long \& A. D. Baddeley (Eds.), Attention and performance $I X$ (pp. 187-203). Hillsdale, NJ: Erlbaum.

Jonides, J., Naveh-Benjamin, M., \& Palmer, J. (1985). Assessing automaticity. Acta Psychologica, 60, 157-171.

Jonides, J., \& Yantis, S. (1988). Uniqueness of abrupt visual onset as an attention-capturing property. Perception \& Psychophysics, 43, 346-354.

Kahneman, D., \& Treisman, A. (1984). Changing views of attention and automaticity. In R. Parasuraman, R. Davies, \& J. Beatty (Eds.), Varieties of attention (pp. 29-61). New York: Academic Press.

Koch, C., \& Ullman, S. (1985). Shifts in selective visual attention: Toward the underlying neural circuitry. Human Neurobiology, 4 , 219-227. 
Krumhansl, C. L. (1982). Abrupt changes in visual stimulation enhance processing of form and location information. Perception \& Psychophysics, 32, 511-523.

Lambert, A., Spencer, E., \& Mohindra, N. (1987). Automaticity and the capture of attention by a peripheral display change. Current Psychological Research \& Reviews, 6, 136-147.

Lennie, P. (1980). Parallel visual pathways: A review. Vision Research, 20, 561-594.

Logan, G. D. (1978). Attention in character-classification tasks: Evidence for the automaticity of component stages. Journal of Experimental Psychology: General, 107, 32-63.

Meyer, D. E., Yantis, S., Osman, A. M., \& Smith, J. E. K. (1985). Temporal properties of human information processing: Tests of discrete versus continuous models. Cognitive Psychology, 17, 445518.

Miller, J. (1989). The control of attention by abrupt visual onsets and offsets. Perception \& Psychophysics, 45, 567-571.

Müller, H. J., \& Rabbitt, P. M. A. (1989). Reflexive and voluntary orienting of visual attention: Time course of activation and resistance to interruption. Journal of Experimental Psychology: Human Perception and Performance, 15, 315-330.

Murphy, T. D., \& Eriksen, C. W. (1987). Temporal changes in the distribution of attention in the visual field in response to precues. Perception \& Psychophysics, 42, 576-586.

Palmer, J., \& Jonides, J. (1988). Automatic memory search and the effects of information load and irrelevant information. Journal of Experimental Psychology: Learning, Memory, and Cognition, 14, 136-144.

Posner, M. I. (1980). Orienting of attention. Quarterly Journal of Experimental Psychology, 32, 3-25.

Posner, M. I., \& Cohen, Y. (1984). Components of visual orienting. In D. Bouma \& D. Bonwhuis (Eds.), Attention and Performance X (pp. 531-556). Hillsdale, NJ: Erlbaum.

Posner, M. I., Cohen, Y., \& Rafal, R. D. (1982). Neural systems control of spatial orienting. Philosophical Transactions of the Royal Society of London, Series B, 298, 187-198.

Posner, M. I., \& Snyder, C. R. R. (1975). Attention and cognitive control. In R. L. Solso (Ed.), Information processing and cognition (pp. 55-85). Hillsdale, NJ: Erlbaum.

Regan, J. E. (1981). Automaticity and learning: Effects of familiarity on naming letters. Journal of Experimental Psychology: Human Perception and Performance, 7, 180-195.

Remington, R., \& Pierce, L. (1984). Moving attention: Evidence for time-invariant shifts of visual selective attention. Perception \& Psychophysics, 35, 393-399.

Schneider, W., \& Fisk, A. D. (1982). Concurrent automatic and controlled visual search: Can processing occur without resource cost? Journal of Experimental Psychology: Learning, Memory, and Cognition, 8, 261-278.

Schneider, W., \& Shiffrin, R. M. (1977). Controlled and automatic human information processing: I. Detection, search, and attention. Psychological Review, 84, 1-66.

Shaw, M. L. (1978). A capacity allocation model for reaction time. Journal of Experimental Psychology: Human Perception and Performance, 4, 586-598.

Shiffrin, R. M., \& Schneider, W. (1977). Controlled and automatic human information processing: II. Perceptual learning, automatic attending, and a general theory. Psychological Review, 84, 127190.

Smith, J. E. K., Yantis, S., \& Meyer, D. E. (1990). Analyses of multinomial mixture distributions: New tests for stochastic models of cognition and action. Submitted for publication.

Todd, J. T., \& Van Gelder, P. (1979). Implications of a transientsustained dichotomy for the measurement of human performance. Journal of Experimental Psychology: Human Perception and Performance, 5, 625-638.

Tolhurst, D. J. (1975). Sustained and transient channels in human vision. Vision Research, 15, 1151-1155.

Townsend, J. T., \& Ashby, F. G. (1983). Stochastic modeling of elementary psychological processes. Cambridge: Cambridge University Press.

Treisman, A. M., \& Gelade, G. (1980). A feature-integration theory of attention. Cognitive Psychology, 12, 97-136.

Yantis, S., \& Johnson, D. N. (in press). Mechanisms of attentional priority. Journal of Experimental Psychology: Human Perception and Performance.

Yantis, S., \& Johnston, J. C. (1990). On the locus of visual selection: Evidence from focused attention tasks. Journal of Experimental Psychology: Human Perception and Performance, 16, 135-149.

Yantis, S., \& Jonides, J. (1984). Abrupt visual onsets and selective attention: Evidence from visual search. Journal of Experimental Psychology: Human Perception and Performance, 10, 601-621.

Received May 24, 1988

Revision received January 9, 1989

Accepted January 10, 1989 